\title{
Age - Related Gastric Changes
}

\author{
Aly Saber ${ }^{1}$, Emad K. Bayumi \\ ${ }^{1}$ Department of general Surgery, Port-Fouad General Hospital, Port-Fouad, Port-Said, Egypt \\ ${ }^{2}$ General Surgery, Crimean Medical Academy Named after S.I. Georgiesky of Crimea Federal University, Crimea, Russia
}

Email address:

Alysaber54@gmail.com (A. Saber), Emadsurg666@hotmail.com (E. K. Bayumi)

\section{To cite this article:}

Aly Saber, Emad K. Bayumi. Age - Related Gastric Changes. Journal of Surgery. Special Issue: Gastrointestinal Surgery: Recent Trends. Vol. 4, No. 2-1, 2016, pp. 20-26. doi: 10.11648/j.js.s.2016040201.15

\begin{abstract}
Aging is a universal process with progressive loss of function accompanied by decreasing fertility and increasing mortality and disability. There are several mechanisms to underlie the primary aging process and probably contribute to agerelated changes in adaptive responses. These mechanisms are oxidative stress, mitochondrial theory, telomeres and cellular senescence, apoptosis and genetic mechanism. There are many physiological changes with aging process including blood pressure, temperature, fever, and composition of body fluids. Age-related changes in bioavailability may be secondary to changes in absorption or gut wall and hepatic metabolism. The stomach lining's capacity to resist damage decreases with age due to alteration of the gastric defense mechanisms and decreased mucosal blood flow. Normal aging is associated with agerelated changes in motor function of the various parts of the gastrointestinal tract such as transit time and gastric emptying. The incidence of many gastrointestinal dysfunctions increases with advancing age that is associated with alterations in the structural and functional integrity of the gastrointestinal tract. The structural changes include mucosa, muscular coat and blood flow. The clinical significance of functional and structural gastric changes may all impact upon gastrointestinal adverse effects and how older people tolerate medicines. Understanding how the upper gastrointestinal tract changes with advancing age could allow interventions that lead to more appropriate prescribing for older people, potentially reduce adverse effects, increase compliance with treatment regimens, and may allow older people to take medications that they would not otherwise tolerate.
\end{abstract}

Keywords: Stomach, Changes, Functional, Structural, Aging

\section{Introduction}

\subsection{Aging Process}

Aging is a universal process whose manifestations are familiar and unambiguous, and these manifestations can be recognized readily after minimal assessment. Aging has been defined as the progressive loss of function accompanied by decreasing fertility and increasing mortality and disability and involves the steady decline of organ functions and the regulation of the body systems. Changes in the regulation of the body systems increase the diversity among older people as with age, the ability of organs to control their normal homeostasis decreases, due to progressive changes in the regulatory mechanisms [1]. These mechanisms are: oxidative Stress, mitochondria, telomeres and cellular senescence and apoptosis.

Increased oxidative stress has been incriminated in physiological conditions, such as aging and exercise, and in several pathological conditions, including cancer, neurodegenerative, cardiovascular diseases, diabetes, inflammatory diseases, and intoxications [2]. Mitochondria are both producers and targets of oxidative stress; this fact forms the basis for the mitochondrial theory of aging. It has been proposed that accumulation of somatic mutations of mitochondrial DNA, induced by exposure to reactive oxygen species generated within mitochondria, leads to errors in the mitochondrial DNA-encoded polypeptides and subsequent defective electron transfer activity and oxidative phosphorylation [3]. DNA damage signals originating from telomeres could be replication independent, and the sensitivity of cells to DNA damage could increase as the overall telomere length declines. The issue of organismal aging as a consequence of short telomeres was raised as a concern and in contrast, nuclear transfer experiments using nuclei from senescent bovine fibroblasts yielded offspring with longer than expected telomeres [1]. Apoptosis seems to be a double-edged sword since during low-level chronic stress, such as in aging, increased resistance to apoptosis can lead to the survival of functionally deficient, post-mitotic 
cells with damaged housekeeping functions. At the molecular level, age-related resistance to apoptosis involves (1) functional deficiency in p53 network, (2) increased activity in the NF- $\mathrm{BB}-\mathrm{IAP} / \mathrm{JNK}$ axis, and (3) changes in molecular chaperones, microRNAs, and epigenetic regulation $[4,5]$.

\subsection{Physiological Changes with Aging in General}

These changes include blood pressure, temperature, fever, and body fluids. The body may also be less likely to start a fever to fight off an infection. The amount and composition of body fluids is reduced too. In older people, the thirst response that normally follows being deprived of water is decreased. The epithelial covering and lining show marked functional and structural changes with age. The skin and mucus membranes, which serve as the primary barriers to infection, may be impaired with age. The lining surfaces of body tissues, which also help prevent infection, may also be compromised, especially in the mouth, urethra, and vagina. Changes occurring in the gastrointestinal and urinary systems are obviously detected. These include the stomach secreting less acid, changes in the way the bowel contracts and outpouchings called diverticular that often form in the bowel lining. There is less change in the gastrointestinal system than in other body system. The muscle contractions of the esophasgus, which affect swallowing, changes, and there is a small amount of atrophy in the lining of the small intestine. The liver decreases in size and blood flow declines. The pancreas drops a little, pancreatic ducts gradually increase in size, and the pancreas atrophies. Gastrointestinal conditions that affect older people include gastrointestinal bleeding, loss of bowel control, peptic ulcer disease, gallbladder disease, diverticulitis, appendicitis, colon and rectal cancers, and hemorrhoids and other rectal problems $[6,7]$.

The cardiovascular system responds less efficiently to stress with age. Physical activity, nutrition, smoking, socioeconomic status, and other lifestyle factors contribute to heart disease in older people. Common heart conditions in older people are high blood pressure, coronary artery disease, diseases of the heart valves, congestive heart failure, and abdominal aortic aneurysm. Blood pressure increases with aging and nearly half of all people over the age of 65 have mild high blood pressure. High blood pressure increases the risk of heart attacks and strokes. Coronary artery disease is the progressive narrowing of the arteries that supply blood to the heart; it is the most common cause of death in people over the age of 65 . Low levels of HDL, or healthy cholesterol, and high levels of LDL, or unhealthy cholesterol, contribute to the development of coronary artery disease that can lead to heart attacks [8].

\section{GIT Functional Changes with Age}

Age-related changes in bioavailability may be secondary to changes in absorption or gut wall and hepatic metabolism $[5,6]$.

\subsection{Gastric Mucosal Defense Mechanisms}

Defense mechanisms permit the gastric mucosa to withstand frequent exposure to damaging factors across a wide range of $\mathrm{pH}$, osmolality, and temperature. These include local defense mechanisms and neurohormonal mechanisms: [9].

\subsubsection{A-Local Gastric Mucosal Defense Mechanisms}

The stomach lining's capacity to resist damage decreases with age, which in turn may increase the risk of peptic ulcer disease, especially in people who use aspirin and other nonsteroidal anti-inflammatory drugs (NSAIDs) and many previous studies have reported an increased susceptibility of the gastric mucosa to various injuries in old animals. Animal and human studies have indicated that aging gastric mucosa has impaired mucosal defenses with decreased mucus and bicarbonate secretion. In the other hand, healthy older people have a reduced mucosal prostaglandin concentration compared with younger individuals. The cyclooxygenase enzyme is involved in this pathway, which is significantly reduced with age. In experimental studies, a reduction in basal gastric blood flow has been demonstrated and the increase in gastric blood flow in response to injury has been shown to be attenuated with advancing age. Moreover, studies have shown that aging gastric mucosa has reduced nitric oxide synthase (NOS) activity and impaired sensory nerve response to luminal acid. In addition, reactive oxygen species (ROS) have been shown to play a role in tissue damage and aging [10].

The mucus-bicarbonate-phospholipid barrier constitutes the first line of mucosal defense and is formed by mucus gel, bicarbonate, and surfactant phospholipids, which cover the mucosal surface. The mucus gel contains phospholipids, and its luminal surface is coated with a film of surfactant phospholipids with strong hydrophobic properties. Mucus gel minimizes luminal loss of $\mathrm{HCO} 3$ - sufficiently to maintain a neutral $\mathrm{pH}$ at the apical cell surfaces. The mucus bicarbonate barrier is the only pre-epithelial barrier between lumen and epithelium and when it is breaks down in disease, the next series of protective mechanisms come into play, including intracellular neutralization of acid, rapid epithelial repair, and maintenance and distribution of mucosal blood flow [11].

The next line of mucosal defense is formed by a continuous layer of surface epithelial cells which secrete mucus and bicarbonate and generate prostaglandins (PGs) and heat shock proteins, and cathelicidins. Because of the presence of phospholipids on their surfaces, these cells are hydrophobic, repelling acid- and water-soluble damaging agents. Interconnected by tight junctions, the surface epithelial cells form a "barrier" preventing back diffusion of acid and pepsin. Aging has been associated with lower capabilities for cellular repair in the gastric mucosa and this reduction is considered secondary to reduced expression of various growth factors such as EGF and TGF- $\alpha$ in the stomach. In the elderly, gastric mucus production, bicarbonate secretion, and gastric mucosal blood flow as well as the gastric prostaglandin biosynthesis are indeed reduced [12].

Mucosal microcirculation is essential for delivery of 
oxygen and nutrients and removal of toxic substances. At the level of the muscularis mucosae, most gastric arteries branch into capillaries, which enter the lamina propria and travel upward in proximity to gastric glandular epithelial cells. At the base of surface epithelial cells, capillaries converge into collecting venules. The endothelial cells lining the microvessels generate potent vasodilators such as nitric oxide (NO) and prostacyclin (PGI2), which protect the gastric mucosa against injury and oppose the mucosal damaging action of vasoconstrictors such as leukotriene $\mathrm{C} 4$, thromboxane A2, and endothelin. PGI2 and NO maintain viability of endothelial cells and prevent platelet and leukocyte adherence to the microvascular endothelial cells, thus preventing compromise of the microcirculation. When the gastric mucosa is exposed to an irritant or when acid back-diffusion occurs, a marked and rapid increase in mucosal blood flow occurs $[1,7,10]$.

Gastric mucosal blood flow may be reduced in the elderly. Recent measurements of basal gastric microcirculation using laser Doppler flowmetry in patients over 65 years of age showed that gastric blood flow is significantly lower than normal. Many investigators have reported a decrease in mucosal blood flow resulting in impairment of gastric mucosal energy metabolism in animal experiments. Recently, endoscopic studies using reflectance spectrophotometry and laser Doppler flowmetry have indicated that gastric mucosal blood flow in human decreases with age. However, changes in energy metabolism in human gastric mucosa with age remains obscure. Impaired angiogenesis in ischemic tissues of old animals was found to be associated with expression of VEGF. Several studies have reported that over-expression of PTEN inhibited cell survival and angiogenesis in the endothelial cells $[10,13]$.

Gastric mucosa and submucosal vessels are innervated by primary afferent sensory neurons and nerves forming a dense plexus at the mucosal base. The nerves fibers from this plexus enter the lamina propria (accompanying capillary vessels) and end just beneath the surface epithelial cells. These nerve endings can sense the luminal content and/or entry of acid into the mucosa via acid-sensing channels. Activation of these nerves directly affects the tone of submucosal arterioles, which regulate mucosal blood flow [13]. Stimulation of gastric sensory nerves leads to the release of neurotransmitters such as calcitonin gene-related peptide and substance $P$ in the nerve terminals located within or in close proximity to the large submucosal vessels. Calcitonin gene-related peptide exerts a mucosal protective action, most likely through vasodilatation of submucosal vessels mediated by NO generation. Interference with any aspect of the sensory innervation, such as ablation of the sensory afferent nerves with chronic, large doses of capsaicin, impairs the hyperemic response and thus diminishes resistance of the gastric mucosa to injury. Moreover, it was suggested that the impaired mucosal defenses and reduced repair in old animals might be due to a decreased density of nerve fibers around the submucosal blood vessels, resulting in reduced activity of sensory neurons leading to an increase of mucosal susceptibility to acid injury $[13,14]$.

\subsubsection{B-Neurohormonal Regulation of Gastric Mucosal Defense}

Gastric mucosal defense is regulated by the central nervous system and hormonal factors. Vagal activation increases mucus gel and surface cell intracellular $\mathrm{pH}$ and central CRF signaling pathways are involved in the endocrine and visceral responses to stress. Peripheral CRFrelated mechanisms also contribute to stress-induced changes in gut motility and mucosal function. The CRF2 receptor is present in the human stomach in which it plays a protective role by inhibiting apoptosis. Various peptides including gastrin, cholecystokinin, thyrotropin-releasing hormone, bombesin, CRF, EGF, peptide YY, neurokinin A analogs, and intragastric peptone exert gastroprotection that is abolished by afferent nerve denervation, blockade of calcitonin generelated peptide receptors, and inhibition of NO synthase. Ghrelin, a peptide hormone produced by $\mathrm{P} / \mathrm{D} 1$ cells in humans, regulates growth hormone secretion at the hypothalamic and pituitary level and elicits orexigenic and appetite stimulating action [15]. Ghrelin also may exert peripheral actions such as gastric mucosal protective and healing action by enhancement of gastric mucosal blood flow via stimulation of NO production and calcitonin gene-related peptide release from sensory afferent nerves. Adrenal glucocorticoids also play an important, facilitating role in gastric mucosal defense. An acute rise of corticosterone during stress is a potent gastroprotective component of the hormonal response to stress. The protective action of glucocorticoids is assumed to occur via their maintenance of glucose homeostasis, gastric blood flow, and mucus secretion and their attenuation of enhanced gastric motility and microvascular permeability $[14,15,16]$.

\subsection{Gastric Secretions}

The stomach consists of three topographic (fundus, corpus, and antrum) and two functional (oxyntic and pyloric gland) areas. The oxyntic gland area, the hallmark of which is the oxyntic or parietal cell, comprises $80 \%$ of the organ (fundus and corpus). The pyloric gland area, the hallmark of which is the gastrin or $\mathrm{G}$ cell, comprises $20 \%$ of the organ (antrum) [17]. Parietal cells secrete hydrochloric acid at a concentration of approximately $160 \mathrm{mmol} / \mathrm{L}$ or $\mathrm{pH} 0.8$. Most studies indicate that the rate of acid secretion by the human stomach changes little with aging unless there is coexisting disease of the oxyntic mucosa such as infection with HP or atrophic gastritis Acid facilitates the digestion of protein and absorption of iron, calcium, and vitamin B-12 as well as prevents bacterial overgrowth and enteric infection. However, when levels of acid (and pepsin) overwhelm mucosal defense mechanisms, ulcers occur. To prevent such damage, gastric acid must be precisely regulated. This is accomplished by a highly coordinated interaction of neural, hormonal, and paracrine pathways. These pathways can be activated directly by stimuli originating in the brain or reflexively by stimuli originating in the stomach such as distension, protein, and 
acid. The principal stimulants of acid secretion are (1) histamine, released from ECL cells (paracrine); (2) gastrin, released from G cells (hormonal); and (3) Acetylcholine (Ach), released from postganglionic enteric neurons (neurocrine). These agents interact with receptors coupled to 2 major signal transduction pathways: adenylate cyclase in the case of histamine and intracellular calcium in the case of gastrin and ACh. The main inhibitor of acid secretion is somatostatin, released from oxyntic and pyloric D cells (paracrine). Each of these agents acts directly on the parietal cell as well as indirectly by modulating the secretion of neuroendocrine cells [18, 19].

Gastric acid secretion is tightly regulated by overlapping neural, hormonal, paracrine, and intracellular pathways in order to achieve the correct amount of acid secretion required by the specific situation. Gastric acid secretion is regulated by biologic agents produced and released by enteroendocrine cells and neurons as well as by exogenously administered substances and infection. Too much acid can lead to gastroesophageal reflux disease, peptic ulcer disease, and stress-related erosion/ulcer disease [20]. Too little acid can interfere with the absorption of certain nutrients, predispose to enteric infection, and interfere with the absorption of some medications. Gastrin, histamine, gastrin-releasing peptide, ghrelin, orexin, and glucocorticoids stimulate whereas leptin, glucagon-like peptide 1, and Helicobacter pylori inhibit acid secretion. The main secretagogues active at the level of the parietal cell are acetylcholine (neurotransmitter), gastrin (hormone), and histamine (paracrine agent); the main inhibitor is somatostatin (paracrine agent). The release of these four agents by neural, hormonal, and paracrine mechanisms and the interactions among them determine the rate of acid secretion in response to physiological stimuli [20, 21]. The antrum and body region of the stomach both have an important role in regulating gastric acid secretion. The antral mucosa contains the $\mathrm{G}$ cells, which release gastrin. This hormone is released in response to ingestion of proteincontaining food and it stimulates the oxyntic mucosa to secrete acid. The hormone acts on the gastrin receptors on the enterochromaffin-like (ECL) cells of the oxyntic mucosa causing them to release histamine, which acts in a paracrine fashion, binding to the H2receptors on the parietal cells and thereby stimulating them to secrete acid. Gastrin also exerts a trophic influence on the ECL cells and parietal cells of the oxyntic mucosa $[18,19]$. Any aging effects on gastric acid secretion are likely to be confounded by Helicobacter pylori eradication and the widespread use of proton pump inhibitors and $\mathrm{H}_{2}$-receptor antagonists among older people. The drugs that require an acidic environment to become ionized (e.g., ketoconazole, ampicillin esters, iron compounds) will be affected most by any age-related changes in gastric acid production. Experimental works showed that aging decreases capacity of the gastric mucosa to secrete acid and pepsin and in aged animals, decreased acid and pepsin output could in part be attributed to mucosal atrophy. The responsiveness of the gastric mucosa to pentagastrin decreases with age [1].

\subsection{Gastric Motility}

Normal aging is associated with important age-related changes in motor function of the various parts of the gastrointestinal tract and some of these important motility changes may occur, either in relation to common age-related disorders, or as a result of certain drugs commonly prescribed in the aged [6-8]. More important is the impact of various age-related diseases on gastrointestinal motility in the elderly: for example, long-standing diabetes mellitus may reduce gastric emptying in up to $50 \%$ of patients; depression significantly prolongs whole-gut transit time; hypothyroidism may prolong oro-caecal transit time; and chronic renal failure is associated with impaired gastric emptying. In addition, various, frequently used drugs in the elderly cause disordered gastrointestinal motility. These drugs include anticholinergics, especially antidepressants with an anticholinergic effect, opioid analgesics and calcium antagonists [22, 23].

\subsection{Gastric Emptying}

Gastric emptying is important as a rate-limiting step in the absorption of orally administered drugs. Gastric emptying is affected by many factors including intragastric $\mathrm{pH}$, intragastric food contents, concomitant disease processes and drug administration [24]. Gastric emptying can be measured in several ways. Early studies included radio-opaque markers and abdominal screening. Later studies utilized radio-isotope markers of both solids and liquids. A safe, noninvasive technique of measuring gastric emptying using real-time ultrasound has been developed. Paracetamol absorption kinetics has been used successfully as a model for gastric emptying of liquids as it is rapidly absorbed from the small bowel but not from the stomach. Heading's study demonstrated a significant correlation between gastric emptying, measured by scintigraphy and time taken to reach maximal plasma paracetamol concentration $\mid 10 \sim$. In the context of drug absorption, paracetamol absorption kinetic parameters are the most relevant in view of the methodological differences. The effect of aging on the motility of the gastrointestinal tract has been reviewed extensively and showed that old age is associated with slowing of gastric emptying, decreased peristalsis, and slowing of colonic transit secondary largely to regionspecific loss of neurons [24, 25].

\section{Structural Changes with Age}

Mucosa of the gastrointestinal (GI) tract is maintained by continuous cell renewal and any deviation in the replicative processes may result in a loss of not only structural but also functional integrity. In general, the incidence of many gastrointestinal dysfunctions, including malignancy increases with advancing age, which in itself is associated with alterations in the structural and functional integrity of the gastrointestinal tract [26]. 


\subsection{Gastric Mucosal Proliferation and Regeneration}

The structural and the functional integrity of the mucosa of various parts of the gastrointestinal tract are maintained by constant renewal of cells and the age-related changes in gastrointestinal mucosal cell proliferation and the regulation of this process at different stages of life are traced by many researchers. In the gastric mucosa, aging is associated with increased activation of certain protein kinases and regulating the expression of a variety of genes that participate in growth related processes. Aging resulted in atrophy of the tissue, as evidenced by the decreased mucosal height and this may be the result of increased cell loss or block in mitotic or other cell cycle regulatory events remains to be determined $[26,27]$.

Age-associated changes in gastrointestinal mucosal cell proliferation could be secondary to alterations in hormonal influences. However, responsiveness of the gastric mucosa to these peptides changes at different stages of life. It was reported that in rats, the gastric mucosa becomes responsive to the growth-promoting action of gastrin around the 3rd postnatal week of life. At this time the parietal cells also become sensitive to gastrin and secrete acid in response to the hormone, when gastrin receptors also appear. On the other hand, the functional properties are either decreased or remain unchanged during advancing age. For example, basal gastric acid and pepsin output decline during aging, as is gastrin secretion. In contrast, antral gastrin levels increase during this period, as is mucosal histidine decarboxylase activity. The age-related decline in gastrin secretion could partly be attributed to a higher ratio of somatostatin (D) to gastrin $(\mathrm{G})$ cells in the antral mucosa. With aging there is also a progressive loss of gastric mucosal responsiveness to both acid secretory and growth promoting actions of gastrin [27]. Gastric mucosal blood flow:

The role of mucosal blood flow in the protection of gastric mucosa is now well recognized. In fact gastric microcirculation supplies both nutrients and bicarbonate to the gastric mucosa and provides removal of back-diffused hydrogen ions. In order to protect the mucosa against acid excess, blood flow normally increases when acid secretion is stimulated by gastric secretagogues. In contrast, most antisecretory agents induce local vasoconstriction secondary to gastric acid inhibition [28]. Blood flow contributes to protection by supplying the mucosa with oxygen and $\mathrm{HCO} 3-$, and by removing $\mathrm{H}+$ and toxic agents diffusing from the lumen into the mucosa. Low mucosal blood flow predisposes to injury, whereas high blood flow protects against injurious agents. Superficial mucosal damage is followed by increased blood flow which supports the healing process and prevents superficial lesions from developing into deep ones [29].

Recently, endoscopic studies using reflectance spectrophotometry and laser Doppler flowmetry have indicated that gastric mucosal blood flow in human decreases with age. In the elderly gastric mucosal defences are impaired. This is in keeping with a reduced gastric prostaglandin biosynthesis and may account for the higher susceptibility of the mucosa to damaging agents. The possible role of atrophic gastritis and Helicobacter pylori infection as independent confounding factors remains to be determined [30].

\subsection{Gastric Mucosa and Muscular Coats}

Whether or not the gastric mucosa undergoes significant changes in normal aging subjects is still open to debate. In normal subjects undergoing endoscopy and evaluated for mucosal thickness, gland number, numbers of parietal, chief and mucous cells at the fundus and of mucopeptic cells at the antrum, with a morphometric method, subgrouping the patients according to their age class [31]. The number of parietal cells tends to increase with age and, on the other hand, the number of mucous cells is reduced in elderly subjects and when considering the parietal-to-mucous cell ratio, this is significantly increased with age. The structure of muscular tunic of human stomach since birth up to the age of 3 months in cadavers showed wideness of groups of smooth myocytes and of stromal gaps between them was measured in cardial and pyloric regions. It was exposed that decrease of wideness of the groups of myocytes and of the stromal gaps accompanied increase of transverse size of the organ. Outstripping development of nonstriated musculature determined rapid growth of stomach region, outstripping changes of stroma determined delayed growth of the region [32].

\section{Clinical Significance of Functional and Structural Gastric Changes}

\subsection{Altered GIT Physiology}

Changes with aging in the gastrointestinal tract are variable, but in some cases they are responsible for a variety of symptoms. Changes in gastrointestinal function can lead to constipation and fecal incontinence as well as changes in gastric emptying play a role in postprandial hypotension. Age-related changes in gastric physiology such as reduced mucosal protection, gastric blood flow and impaired repair mechanisms may all impact upon gastrointestinal adverse effects and how older people tolerate medicines $[6,7,8]$.

\subsection{Age-related Changes in the Physiology of Drug Bioavailability}

The bioavailability of any drug after oral administration depends upon many factors, including the fraction of the administered dose absorbed through the gastrointestinal mucosa and the fraction of the absorbed dose that passes through the gastrointestinal tract into the hepatic portal blood unmetabolized [1]. Old age is associated with slowing of gastric emptying, decreased peristalsis, and slowing of colonic transit secondary largely to region-specific loss of neurons $[1,6,7]$.

\subsection{Other Gastrointestinal Pathology}

Diseases of the upper gastrointestinal tract such as peptic 
ulceration and gastric cancer become more common and more severe with advancing age. In the normal stomach and duodenum, there is a balance between mucosal protective mechanisms and endogenous (gastric acid and pepsin) and exogenous aggressive factors. The high incidence of gastrointestinal pathology seen in older age groups is not related to increase in the secretion of endogenous aggressive factors. Recent work suggests that gastrointestinal mucosal protective mechanisms are impaired with age. The roles in the gastrointestinal tract of molecules that have been implicated in mucosal repair, such as trefoil peptides and matrix components, are beginning to be elucidated and their study in older people is essential to ensure appropriate, efficient, cost-effective management of gastric pathology in the elderly. Strategies to improve the management of upper gastrointestinal diseases in older people will reduce mortality and improve quality of life [33].

\section{Conclusion}

Aging is associated with significant alterations in both gastric functional and structural patterns. All the functional activities of the stomach show marked changes with age such as secretory and motor functions. Structural gastric alterations involve all the histological layers, mucosa, submucoca and muscular coat.

\section{References}

[1] McLean AJ\& Le Couteur DG. Aging biology and geriatric clinical pharmacology. Pharmacol Rev. 2004; 56(2): 163-84.

[2] Preiser, JC. Oxidative StressJPEN J Parenter Enteral Nutr, (2012) 36: (2) 147-154.

[3] Collins Y, Chouchani ET, James AM, Menger KE, Cochemé HM, Murphy MP. Mitochondrial redox signalling at a glance. J Cell Sci. 2012 15; 125(Pt 4): 801-6.

[4] Shen J, Tower J. Programmed cell death and apoptosis in aging and life span regulation. Discov Med. 2009; 8(43): 223-6.

[5] Warner HR. Aging and regulation of apoptosis. Curr Top Cell Regul. 1997; 35: 107-21. Review.

[6] Saber A, Gad MA, Ellabban GM. Perforated duodenal ulcer in high risk patients: Is percutaneous drainage justified? North Am J Med Sci 2012; 4: 35-9.

[7] Saber A. (2011). Perforated Duodenal Ulcer in High Risk Patients, Peptic Ulcer Disease, Jianyuan Chai (Ed.), ISBN: 978-953-307-976-9, InTech, Available from: http://www.intechopen.com/articles/show/title/perforatedduodenal-ulcer-in-high-risk-patients.

[8] Saber A. Perioperative Care of Elderly Surgical Patients. American Medical Journal, 2013: 4 (1): 63-77.

[9] El-Sayed EA, Mosaad M, Wasfy A and Aref W. Age Related Changes in Gastric Function and Histopathology of the Gastric Mucosa. Arab J. Gastro, 2005, 6, (2): 119-123.
[10] Laine L, Takeuchi K, Tarnawski A. Gastric mucosal defense and cytoprotection: bench to bedside. Gastroenterology. 2008; 135(1): 41-60.

[11] Ham M, Kaunitz JD. Gastroduodenal defense. Curr Opin Gastroenterol. 2007; 23: 607-616.

[12] Allen A, Flemström G. Gastroduodenal mucus bicarbonate barrier: protection against acid and pepsin. Am J Physiol Cell Physiol. 2005; 288: C1-C19.

[13] Holzer P. Role of visceral afferent neurons in mucosal inflammation and defense. Curr Opin Phamacol. 2007; 7: 563-569.

[14] Gyires K, Toth VE, Zadori ZS. Gastric mucosal protection: from the periphery to the central nervous system. J Physiol Pharmacol. 2015 Jun; 66(3): 319-29.

[15] Ham M, Kaunitz JD. Gastroduodenal defense. Curr Opin Gastroenterol. 2007; 23: 607-616.

[16] Taché Y. Brainstem Neuropeptides and Vagal Protection of the Gastric Mucosal Against Injury: Role of Prostaglandins, Nitric Oxide and Calcitonin-Gene Related Peptide in Capsaicin Afferents. Curr Med Chem. 2012; 19(1): 35-42.

[17] Joseph IMP, Zavros Y, Merchant JL, et al. A model for integrative study of human gastric acid secretion. J Appl Physiol. 2003; 94: 1602-1618.

[18] Schubert ML, Peura DA. Control of Gastric Acid Secretion in Health and Disease. Gastroenterology. 2008 Jun; 134(7): 1842-60.

[19] Piqueras L, Martínez V. Role of somatostatin receptors on gastric acid secretion in wild-type and somatostatin receptor type 2 knockout mice. Naunyn Schmiedebergs Arch Pharmacol. 2004 Dec; 370(6): 510-20.

[20] Schubert ML. Gastric secretion. Curr Opin Gastroenterol. 2007 Nov; 23(6): 595-601.

[21] Schubert ML. Gastric secretion. Curr Opin Gastroenterol. 2014 Nov; 30(6): 578-82.

[22] Bitar K, Greenwood-Van Meerveld B, Saad R, Wiley JW.Aging and gastrointestinal neuromuscular function: insights from within and outside the gut. Neurogastroenterol Motil (2011) 23, 490-501.

[23] Salles N. Basic mechanisms of the aging gastrointestinal tract. Dig Dis 2007; 25: 112-7.

[24] Inui A, Asakawa A, Bowers CY, Mantovani G, Laviano A, Meguid MM. Fuiimiva M. Ghrelin, appetite, and gastric motility: the emerging role of the stomach as an endocrine organ. FASEB J. 2004 Mar; 18(3): 439-56.

[25] Soenen S, Rayner CK, Horowitz M, Jones KL. Gastric Emptving in the Elderly. Clin Geriatr Med. 2015 Aug; 31(3): 339-53.

[26] Vilkova IV. Structural changes in the stomach muscular coat as basis for heterochronia in human early postnatal ontogenesis. Morfologiia. 2001; 119(3): 80-3.

[27] Ishihara M, Ito M. Influence of aging on gastric ulcer healing activities of cimetidine and omeprazole. Eur J Pharmacol. 2002 May 31; 444(3): 209-15.

[28] Wallace JL. Prostaglandins, NSAIDs, and Gastric Mucosal Protection: Why Doesn't the Stomach Digest Itself? Physiological Reviews Published, 2008; 88 (4): 1547-1565. 
[29] Yandrapu H, Sarosiek J. Protective Factors of the Gastric and Duodenal Mucosa: An Overview. Curr Gastroenterol Rep. 2015 Jun; 17(6): 24. doi: 10.1007/s11894-015-0452-2.

[30] Tarnawski AS, Ahluwalia A, Jones MK. Increased susceptibility of aging gastric mucosa to injury: the mechanisms and clinical implications. World J Gastroenterol. 2014 Apr 28; 20(16): 4467-82.

[31] El Shall LM. Age Related Changes in the Fundic Mucosa of Stomach of Male Albino Rats (Histological and Immunohistochemical Study). Egyp J Histology, 2010, 33 (1): $32-44$.
[32] Farinati F, Formentini S, Della Libera G, Valiante F, Fanton MC, Di Mario F, Vianello F, Pilotto A, Naccarato R. Changes in parietal and mucous cell mass in the gastric mucosa of normal subjects with age: a morphometric study. Gerontology. 1993; 39(3): 146-51.

[33] Franceschi M, Di Mario F, Leandro G, Maggi S, Pilotto A. Acid-related disorders in the elderly. Best Pract Res Clin Gastroenterol. 2009; 23(6): 839-48. 